Punjab University Journal of Mathematics

(ISSN 1016-2526)

Vol. 53(2)(2021) pp. 19-33

\title{
A Study of Completely Inverse Paramedial AG-Groupoids
}

\author{
Muhammad $\operatorname{Rashad}^{a}, \operatorname{Imtiaz}_{\mathrm{Ahmad}^{b}}$ and Faruk Karaaslan ${ }^{c, *}$ \\ ${ }^{a, b}$ Department of Mathematics, University of Malakand, Chakdara, Pakistan. \\ Email:rashad@uom.edu.pk,iahmad@uom.edu.pk \\ ${ }^{c}$ Department of Mathematics, Cankırı Karatekin University \\ Email: fkaraaslan@karatekin.edu.tr
}

Received: 25 Feburary, 2020 / Accepted: 20 January, 2021 / Published online: 22 Feburary, 2021

\begin{abstract}
A magma $S$ that meets the identity, $x y \cdot z=z y \cdot x, \forall x, y, z \in S$ is called an AG-groupoid. An AG-groupoid S gratifying the paramedial law: $u v \cdot w x=x v \cdot w u, \forall u, v, w, x \in S$ is called a paramedial AGgroupoid. Every AG-grouoid with a left identity is paramedial. We extend the concept of inverse AG-groupoid $[4,7]$ to paramedial AG-groupoid and investigate various of its properties. We prove that inverses of elements in an inverse paramedial AG-groupoid are unique. Further, we initiate and investigate the notions of congruences, partial order and compatible partial orders for inverse paramedial AG-groupoid and strengthen this idea further to a completely inverse paramedial AG-groupoid. Furthermore, we introduce and characterize some congruences on completely inverse paramedial AG-groupoids and introduce and characterize the concept of separative and completely separative ordered, normal sub-groupoid, pseudo normal congruence pair, and normal congruence pair for the class of completely inverse paramedial AG-groupoids. We also provide a variety of examples and counterexamples for justification of the produced results.
\end{abstract}

\begin{abstract}
AMS (MOS) Subject Classification Codes: 20N02; 08A30
Key Words: Completely inverse AG-groupoids, paramedial AG-groupoids, congruences, natural partial order.

\section{INTRODUCTION}

The theory of AG-groupoid is introduced in 1972 by Kazim and Naseer [6]. AGgroupoids generalize the class of commutative semigroups and satisfies the medial law, $a b \cdot c d=a c \cdot b d$. Throughout this article, $S$ will represent an AG-groupoid otherwise stated else. This structure is closely related to a commutative semigroup because a commutative AG-groupoid is always associative [7]. An AG-groupoid may or may not contains a left identity element, and if an AG-groupoid contains a left identity, then this left identity is
\end{abstract}


unique. It is important to mention here that if an AG-groupoid contains identity or even a right identity element, then it becomes a commutative monoid. Further, the left identity of an AG-groupoid permits inverses of elements in the structure. An AG-groupoid with the left identity is called AG-monoid, and satisfies the paramedial property, $a b \cdot c d=d b \cdot c a$. Every paramedial AG-groupoid also satisfies the bi-commutative property,

$$
a b \cdot c d=d c \cdot b a \forall, a, b, c, d .
$$

AG-groupoid $S$ with the property $a b \cdot c=b \cdot a c$ is called $\mathrm{AG}^{*}$ and is called $\mathrm{AG}^{* *}$ if it satisfies the identity $a \cdot b c=b \cdot a c$. We shall use the juxtaposition to avoid excessive parenthesization and dots i.e. $u v$ will mean $u \cdot v, u v \cdot w t$ for $(u \cdot v)(w \cdot t)$, and $(u v \cdot w) t$ for $((u \cdot v) w) t$. AG-groupoid is a non-associative structure in general that possess a variety of applications in the field of flock theory, geometry and finite mathematics $[10,11,12,13]$. Fuzzification of the field has made it more interesting and applicable [1, 5, 14, 15].

Various other aspects of the said structure are also investigated by different researchers in a variety of papers $[16,17,18,19,20]$ and the references therein. Inverse and completely inverse AG-groupoids are defined by Mushtaq and Iqbal [7], Peter V. Protic [3] and Wieslaw A. Dudek and Roman S. Gigon [4]. Some congruences on an inverse and completely inverse $\mathrm{AG}^{* *}$-groupoids are defined $[2,3,4,8,21]$. In this section we define some congruences on completely inverse paramedial AG-groupoid. To proceed further, we start with the following definition.

Definition 1.1. [4, 7] An AG-groupoid $S$ is called inverse AG-groupoid, if for every $u \in S$, there exists $u^{\prime} \in S$ such that $u=u u^{\prime} \cdot u$ and $u^{\prime} u \cdot u^{\prime}=u^{\prime}$. By $u^{\prime}$ we mean the inverse of u. An AG-groupoid $S$ is called completely inverse AG-groupoid if it satisfies the identity $u u^{\prime}=u^{\prime} u$ for all $u \in S$.

It is proved by $Q$. Mushtaq and M. Iqbal [7] that if $u^{\prime}$ is an inverse of $u$ and $v^{\prime}$ is an inverse of $v$ in an AG-groupoid, then

$$
(u v)^{\prime}=u^{\prime} v^{\prime}
$$

Example 1.2. Consider AG-groupoid $S=\{1,2,3,4\}$ defined in Table 1. Then, the relation $\leq$ define as $a \leq b \Longleftrightarrow a=a a^{-1} \cdot b$ is compatible on AG-groupoid $S$.

\begin{tabular}{c|llll}
$\cdot$ & 1 & 2 & 3 & 4 \\
\hline 1 & 1 & 2 & 3 & 4 \\
2 & 2 & 1 & 4 & 3 \\
3 & 4 & 3 & 2 & 1 \\
4 & 3 & 4 & 1 & 2
\end{tabular}

Table 1

Definition 1.3. An AG-groupoid $(S, \cdot)$ is called an ordered $A G$-groupoid, if $S$ posses an order. In this case, we can write $(S, \cdot, \leq)$.

Definition 1.4. An ordered AG-groupoid $(S, \cdot, \leq)$ is called separative if

(1) $\forall u, v \in S, u^{2} \leq u v, v u \leq v^{2} \Rightarrow u \leq v$.

(2) $\forall u, v \in S, u^{2} \leq v u, u v \leq v^{2} \Rightarrow u \leq v$.

In Example (1.2) the relation, $\leq$ is separative. 
Definition 1.5. A separative ordered $A G$-groupoid $S$ is called completely separative if

$$
u, v, x, y \in S, x \leq y,(x y) u \leq(x y) v \Rightarrow x^{2} u \leq x^{2} v, y^{2} u \leq y^{2} v .
$$

\section{CONGRUENCES}

In this section we define some relations on paramedial and inverse paramedial AGgroupoid $S$. We prove that the following relations are congruences on paramedial and inverse paramedial AG-groupoid $S$.

(1) $\eta=\{(u, v) \in S \times S:(\exists l \in E(S)), l u=l v\}$;

(2) $\mu=\{(u, v) \in S \times S: x u=x v, \forall x \in S\}$;

(3) $\rho=\left\{(u, v) \in S \times S: u^{-1} u=v^{-1} v\right\}$.

Here $E(S)$ denotes the set of idempotent elements of $S$.

Remark 2.1. Let $S$ be a paramedial $A G$-groupoid, and $g_{1}, g_{2} \in E(S)$. Then by paramedial and medial law,

$$
g_{1} g_{2}=g_{1} g_{1} \cdot g_{2} g_{2}=g_{2} g_{1} \cdot g_{2} g_{1}=g_{2} g_{2} \cdot g_{1} g_{1}=g_{2} g_{1} .
$$

It follows that $E(S)$ is a semilattice.

The inverses in an inverse paramedial AG-grupoid are unique as proved in the following.

Remark 2.2. Let $S$ be an inverse paramedial AG-groupoid, and $a, b \in V(u)$. Then

$$
\begin{aligned}
u a & =(u b \cdot u) a=a u \cdot u b \quad \text { (by the left invertive law) } \\
& =b u \cdot u a \quad \text { (by the paramedial property) } \\
& =(u a \cdot u) b=u b \quad \text { (by the left invertive law) } \\
\Rightarrow u a & =u b .
\end{aligned}
$$

Thus

$$
\begin{aligned}
a & =a u \cdot a=(a u(a u \cdot a)) \quad(\text { by medial law }) \\
& =(a \cdot a u)(u a) \quad(\text { by medial law }) \\
& =(a \cdot a u)(u b) \quad(\text { by 2.2) } \\
& =(b \cdot a u)(u a) \quad(\text { by paramedial property) } \\
& =(b \cdot a u)(u b) \quad(\text { by 2.2) } \\
& =(b u)(a u \cdot b) \quad(\text { by medial law }) \\
& =(b u)(b u \cdot a) \quad(\text { by left invertive law }) \\
& =(b \cdot b u)(u a) \quad(\text { by medial law }) \\
& =(b \cdot b u)(u b) \quad(\text { by 2.2) } \\
& =(b u)(b u \cdot b) \quad(\text { by medial law }) \\
& =b u \cdot b=b . \\
\Rightarrow a & =b .
\end{aligned}
$$

It follows that $|V(u)|=1$, and the inverse of $u \in S$ is unique. We shall denote it by $u^{-1}$.

Theorem 2.3. Let $S$ be a paramedial $A G$-groupoid and $E(S) \neq \emptyset$. Then the relation $\eta$ defined on $S$ in Section 2 Part (1) is a congruence relation on $S$. 
Proof. Clearly, the relation $\eta$ is a reflexive and symmetric on $E(S) \neq \emptyset$. In order to prove transitivity of $\eta$ let $u \eta v, v \eta w$. Then $l u=l v, m v=m w$ for some $l, m \in E(S)$. Now by the left invertive, paramedial, medial laws and the assumption, we have

$$
\begin{array}{rl}
(l m) u=(l l \cdot m) u=u m \cdot l l & =l m \cdot l u=l m \cdot l v= \\
=l l \cdot m v=l l \cdot m w=w l \cdot m l & = \\
l & w m \cdot l l=w m \cdot l=l m \cdot w .
\end{array}
$$

Thus $l m \cdot u=l m \cdot w$. Since $l m \in E(S)$, so $u \eta w$ equivalently $\eta$ is transitive. Thus $\eta$ is an equivalence relation. Now let $u \eta v, w \in S$. Then $l u=l v$ for some $l \in E(S)$, and

$$
l(u w)=l l \cdot u w=l u \cdot l w=l v \cdot l w=l l \cdot v w=l(v w) \Rightarrow u w \eta v w .
$$

Similarly, wu$\eta w v$. Thus $\eta$ is compatible and hence is a congruence on $S$. Hence the result proved.

Theorem 2.4. Let $S$ be a paramedial AG-groupoid. Then the relation $\mu$ defined on $S$ with

$$
\mu=\{(u, v) \in S \times S: \quad x u=x v, \forall x \in S\}
$$

is a congruence relation on $S$.

The following result holds for a more general class of inverse paramedial AG-groupoid.

Theorem 2.5. Let $S$ be an inverse AG-groupoid. Then the relation $\rho$ defined on $S$ with

$$
\rho=\left\{(u, v) \in S \times S: \quad u^{-1} u=v^{-1} v\right\}
$$

is a congruence relation on $S$.

Proof. Clearly, $\rho$ is an equivalence relation. Now for left compatibility, let $u, v, w \in S$ such that $u \rho v$. Then we have

$$
\begin{aligned}
(w u)^{-1}(w u) & =\left(w^{-1} u^{-1}\right)(w u) \\
& =\left(w^{-1} w\right)\left(u^{-1} u\right) \\
& =\left(w^{-1} w\right)\left(v^{-1} v\right) \\
& =\left(w^{-1} v^{-1}\right)(w v) \\
& =(w v)^{-1}(w v) \\
\Rightarrow(w u)^{-1}(w u) & =(w v)^{-1}(w v) \Rightarrow u \rho v \Rightarrow w u \rho w v .
\end{aligned}
$$

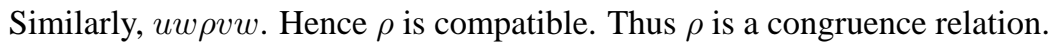

Example 2.6. Consider Example (1.2), then the relation $\rho$ defined in Equation (2.3) is given as under,

$$
\begin{aligned}
\rho= & \{(1,1),(1,2),(1,3),(1,4),(2,1),(2,2),(2,3),(2,4), \\
& (3,1),(3,2),(3,3),(3,4)(4,1),(4,2),(4,3),(4,4)\}
\end{aligned}
$$

is a congruence relation.

Similarly, for Table 2 of an AG-groupoid $(S, \cdot)$ the $\rho$ defined in Equation (2.3) as under is a congruence relation on $S$.

$$
\rho=\{(1,1),(2,2),(3,3),(4,4),(5,5)\}
$$




\begin{tabular}{c|ccccc}
$\cdot$ & 1 & 2 & 3 & 4 & 5 \\
\hline 1 & 1 & 3 & 2 & 5 & 4 \\
2 & 4 & 5 & 3 & 1 & 2 \\
3 & 5 & 2 & 4 & 3 & 1 \\
4 & 3 & 4 & 1 & 2 & 5 \\
5 & 2 & 1 & 5 & 4 & 3
\end{tabular}

Table 2

\section{NATURAL PARTIAL ORDER}

Here we discuss natural partial relation on an inverse paramedial AG-groupoid $S$ and investigate some of its properties. We start with the following theorem.

Theorem 3.1. Let $S$ be an inverse paramedial AG-groupoid. Then the relation $\leq$,

$$
u \leq v \Leftrightarrow u=u u^{-1} \cdot v
$$

is a partial order relation and is compatible on $S$.

Proof. The relation $\leq$ is clearly reflexive as $S$ is inverse paramedial AG-groupoid. $\leq$ is anti-symmetric: Assume that $u \leq v$ and $v \leq u$, then $u=u u^{-1} \cdot v$ and $v=v v^{-1} \cdot u$. Thus by assumption, left invertive, paramedial and medial laws,

$$
\begin{aligned}
u=u u^{-1} \cdot v & =\left(\left(u u^{-1} \cdot v\right) u^{-1}\right)\left(v v^{-1} \cdot u\right) \\
& =\left(u^{-1} v \cdot u u^{-1}\right)\left(v v^{-1} \cdot u\right) \\
& =\left(u^{-1} v \cdot v v^{-1}\right)\left(u u^{-1} \cdot u\right) \\
& =\left(v^{-1} v \cdot v u^{-1}\right)\left(u u^{-1} \cdot u\right) \\
& =\left(v^{-1} v \cdot u u^{-1}\right)\left(v u^{-1} \cdot u\right) \\
& =\left(v^{-1} v \cdot u u^{-1}\right)\left(u u^{-1} \cdot v\right) \\
& =\left(v^{-1} v \cdot u u^{-1}\right)(u) \\
& =\left(u \cdot u u^{-1}\right)\left(v^{-1} v\right) \\
& =\left(u v^{-1}\right)\left(u u^{-1} \cdot v\right) \\
& =\left(v v^{-1}\right)\left(u u^{-1} \cdot u\right) \\
& =v v^{-1} \cdot u \\
& =v .
\end{aligned}
$$

Thus $u=v$. Hence $\leq$ is anti-symmetric.

$\leq$ is transitive: To this end, assume that $u \leq v$ and $v \leq w$ this gives that

$$
\begin{aligned}
u & =u u^{-1} \cdot v \\
\text { and } \quad v & =v v^{-1} \cdot w
\end{aligned}
$$


Now, using Equations (3.5) and (3.6), left invertive law, medial law, paramedial law and reflexive property, we have

$$
\begin{aligned}
u & =u u^{-1} \cdot v=\left(u u^{-1}\right)\left(v v^{-1} \cdot w\right) \\
& =\left(\left(u u^{-1} \cdot v\right) u^{-1}\right)\left(v v^{-1} \cdot w\right) \\
& =\left(u^{-1} v \cdot u u^{-1}\right)\left(v v^{-1} \cdot w\right) \\
& =\left(u^{-1} v \cdot v v^{-1}\right)\left(u u^{-1} \cdot w\right) \\
& =\left(\left(v v^{-1} \cdot v\right) u^{-1}\right)\left(u u^{-1} \cdot w\right) \\
& =\left(v u^{-1}\right)\left(u u^{-1} \cdot w\right) \\
& =\left(w u^{-1}\right)\left(u u^{-1} \cdot v\right) \\
& =w u^{-1} \cdot u \\
& =u u^{-1} \cdot w \\
\Rightarrow u & \leq w .
\end{aligned}
$$

Equivalently, $\leq$ is transitive, and thus the relation $\leq$ is a partial order on $S$. Next, for left compatibility, assume that $u \leq v$ and $w \in S$. Then

$$
\begin{aligned}
w u=w\left(u u^{-1} \cdot v\right) & =\left(w w^{-1} \cdot w\right)\left(u u^{-1} \cdot v\right) \\
& =\left(w w^{-1} \cdot u u^{-1}\right)(w v) \\
& =\left(w u \cdot w^{-1} u^{-1}\right) w v \\
& =\left(w u \cdot(w u)^{-1}\right) w v \Rightarrow w u \leq w v .
\end{aligned}
$$

Hence the relation $\leq$ is left compatible. Further,

$$
\begin{aligned}
u w=\left(u u^{-1} \cdot v\right) w=\left(u u^{-1} \cdot v\right)\left(w w^{-1} \cdot w\right) & \\
& =\left(u u^{-1} \cdot w w^{-1}\right)(v w) \\
& =\left(u w \cdot u^{-1} w^{-1}\right) v w \\
& =\left(u w \cdot(u w)^{-1}\right) v w \Rightarrow u w \leq v w .
\end{aligned}
$$

Thus the relation $\leq$ is right compatible, and whence is compatible.

It is illustrated in the following that the relation $\leq$ defined on an inverse paramedial AG-grupoid is a compatible partial order.

Example 3.2. See Example (1.2), the partial order $\leq$ as defined with ( 3. 4 ) and given below, is a compatible partial order on AG-groupoid $S$.

$$
\leq=\{(1,1),(2,2),(3,3),(4,4)\}
$$

Corollary 3.3. Let $S$ be an inverse paramedial AG-groupoid and $u, v \in S$. Then $u \leq v \Leftrightarrow$ $u u^{-1}=v u^{-1}$ 
Proof. Let $u \leq v$. Then

$$
\begin{array}{rll}
u u^{-1} & =\left(u u^{-1} \cdot v\right) u^{-1} \quad(\text { By Theorem 3.1) } \\
& =\left[\left(u u^{-1}\right)\left(v v^{-1} \cdot v\right)\right] u^{-1} \quad \text { (By Theorem 3.1) } \\
& \left.=\left[\left(v \cdot v v^{-1}\right)\left(u^{-1} u\right)\right] u^{-1}\right) \quad \text { (By bi-commutative) } \\
& =\left(u^{-1} \cdot u^{-1} u\right)\left(v \cdot v v^{-1}\right) \quad \text { (By left invertive law) } \\
& =\left(v v^{-1} \cdot v\right)\left(u^{-1} u \cdot u^{-1}\right) \quad(\text { (By bi-commutative) }) \\
& =v u^{-1} \quad(\text { By Theorem 3.1). }
\end{array}
$$

Conversely, let $u, v \in S$. Then

$$
u u^{-1}=v u^{-1} \Rightarrow u u^{-1} \cdot u=v u^{-1} \cdot u \Rightarrow u=u u^{-1} \cdot v .
$$

Thus $u \leq v$. Hence the result is proved.

In inverse AG-groupoid $u u^{-1}$ and $u^{-1} u$ are not necessarily idempotent as shown in Table 3 and Table 4.

\begin{tabular}{c|cccc}
$*$ & 1 & 2 & 3 & 4 \\
\hline 1 & 2 & 2 & 4 & 4 \\
2 & 2 & 2 & 2 & 2 \\
3 & 1 & 2 & 3 & 4 \\
4 & 1 & 2 & 1 & 2
\end{tabular}

Table 3

\begin{tabular}{c|cccc}
$\cdot$ & 1 & 2 & 3 & 4 \\
\hline 1 & 2 & 3 & 1 & 4 \\
2 & 4 & 1 & 3 & 2 \\
3 & 3 & 2 & 4 & 1 \\
4 & 1 & 4 & 2 & 3
\end{tabular}

Table 4

In the above Table $3,(S, *)$ is an inverse AG-groupoid such that the inverses of $1,2,3,4$ are $4,2,3,1$ respectively. Clearly, $(1 * 4) *(1 * 4) \neq 1 * 4$. Similarly, $(S, \cdot)$ in Table 4 is an inverse AG-groupoid such that the inverses of $1,2,3,4$ are $4,3,2,1$ respectively. Clearly, $(1 \cdot 2) \cdot(1 \cdot 2) \neq 1 \cdot 2$. However, in completely inverse AG-groupoid $u u^{-1}$ and $u^{-1} u$ are idempotents, which is proved in Lemma 4.1.

\section{NORMAL CONGRUENCE PAIR}

Let $S$ denotes a completely inverse paramedial AG-grupoid in which in which we have $u u^{-1}=u^{-1} u$ or equivalently $u u^{-1}, u^{-1} u \in E(S)$ holds for each $u \in S$. Then the following lemma holds.

Lemma 4.1. Let $S$ be an inverse paramedial $A G$-groupoid, $u \in S$. Then

$$
u u^{-1}, u^{-1} u \in E(S) \Leftrightarrow u u^{-1}=u^{-1} u .
$$

Proof. Let $u u^{-1}=u^{-1} u$. Then

$$
\left(u u^{-1}\right)^{2}=u u^{-1} \cdot u u^{-1}=u^{-1} u \cdot u u^{-1}=\left(u u^{-1} \cdot u\right) u^{-1}=u u^{-1} .
$$


Conversely, let $u u^{-1}, u^{-1} u \in E(S)$. Then

$$
\begin{aligned}
u u^{-1} & =u u^{-1} \cdot u u^{-1} \\
& =\left(u u^{-1} \cdot u u^{-1}\right) u u^{-1} \\
& =\left(\left(u u^{-1} \cdot u^{-1}\right) u\right) u u^{-1} \\
& =\left(u u^{-1} \cdot u\right)\left(u u^{-1} \cdot u^{-1}\right) \\
& =\left(u^{-1} u\right)\left(u u^{-1} \cdot u u^{-1}\right) \\
& =\left(u^{-1} u\right)\left(\left(u u^{-1} \cdot u^{-1}\right) u\right) \\
& =\left(u^{-1} u\right)\left(\left(u u^{-1} \cdot u^{-1}\right)\left(u u^{-1} \cdot u\right)\right) \\
& =\left(u^{-1} u\right)\left(\left(u u^{-1} \cdot u u^{-1}\right)\left(u^{-1} u\right)\right) \\
& =\left(u^{-1} u\right)\left(u u^{-1} \cdot u^{-1} u\right) \\
& =\left(u^{-1} u\right)\left(\left(u^{-1} u \cdot u^{-1}\right) u\right) \\
& =\left(u^{-1} u\right)\left(u^{-1} u\right) \\
& =u^{-1} u .
\end{aligned}
$$

Hence the lemma is proved.

The following is a consequence of Theorem (3.1)

Corollary 4.2. Let $S$ be a completely inverse paramedial $A G$-groupoid and $u, v \in S$. Then $u \leq v \Leftrightarrow(\exists g \in E(S)) u=g v$.

Proof. Let $u, v \in S$. Then $u \leq v$ if and only if, $u=u u^{-1} \cdot v$. Since $u u^{-1} \in E(S)$, therefore if $g=u u^{-1}$ then $u=g v$.

Conversely, let $u, v \in S$ be such that $g \in E(S)$ and $u=g v$. Since $u u^{-1}=u^{-1} u \in E(S)$ and $E(S)$ is a semi-lattice, we have

$$
\begin{aligned}
u u^{-1} \cdot v & =\left(g v \cdot g v^{-1}\right) v \\
& =\left(g v \cdot g v^{-1}\right)\left(v v^{-1} \cdot v\right) \\
& =\left(g v \cdot v v^{-1}\right)\left(g v^{-1} \cdot v\right) \\
& =\left(\left(v v^{-1} \cdot v\right) g\right)\left(v v^{-1} \cdot g\right) \\
& =(v g)\left(v v^{-1} \cdot g\right) \\
& =(g g)\left(v v^{-1} \cdot v\right) \\
& =g v \Rightarrow u u^{-1} \cdot v \\
& =u .
\end{aligned}
$$

Thus for each $u, v, x, y \in S$, we have $x u \leq x v \Rightarrow u x \leq v x$ and so $u \leq v$.

Lemma 4.3. Let $(S, \cdot, \leq)$ be a separative order inverse paramedial AG-groupoid. Then for each $u, v, x, y \in S$ we have,

(1) $x u \leq x v \Leftrightarrow u x \leq v x$,

(2) $x^{2} u \leq x^{2} v \Leftrightarrow x u \leq x v$.

Proof. Let $u, v \in S$. Then 
(1) $x u \leq x v$. Since $x u \cdot x u \leq x v \cdot x u$, we have

$$
\begin{aligned}
x u \leq x v & \Rightarrow x u \cdot x u \leq x v \cdot x u \\
& \Rightarrow u u \cdot x x \leq u v \cdot x x \\
& \Rightarrow u x \cdot u x \leq u v \cdot x x \\
& \Rightarrow(u x)^{2} \leq u x \cdot v x .
\end{aligned}
$$

Also

$$
\begin{aligned}
x u \leq x v & \Rightarrow x u \cdot x v \leq x v \cdot x v \\
& \Rightarrow v u \cdot x x \leq v v \cdot x x \\
& \Rightarrow v x \cdot u x \leq v x \cdot v x \\
& \Rightarrow v x \cdot u x \leq(v x)^{2}
\end{aligned}
$$

Similarly, $x u \leq x v \Rightarrow(u x)^{2} \leq v x \cdot u x$ and $u x \cdot v x \leq(v x)^{2}$. Hence $u x \leq v x$.

(2) $x^{2} u \leq x^{2} v$. Since $x^{2} u \cdot u \leq x^{2} v \cdot u$, we have

$$
\begin{aligned}
x^{2} u \leq x^{2} v \Rightarrow x^{2} u \cdot u \leq x^{2} v \cdot u & \\
& \Rightarrow u u \cdot x^{2} \leq u v \cdot x^{2} \\
& \Rightarrow u x \cdot u x \leq u x \cdot v x \\
& \Rightarrow(u x)^{2} \leq u x \cdot v x .
\end{aligned}
$$

Also

$$
\begin{aligned}
x^{2} u \leq x^{2} v \Rightarrow x^{2} u \cdot v \leq x^{2} v \cdot v & \\
& \Rightarrow v u \cdot x^{2} \leq v v \cdot x^{2} \\
& \Rightarrow v x \cdot u x \leq v x \cdot v x \\
& \Rightarrow v x \cdot u x \leq(v x)^{2}
\end{aligned}
$$

Similarly, $x^{2} u \leq x^{2} v \Rightarrow(u x)^{2} \leq v x \cdot u x$ and $u x \cdot v x \leq(v x)^{2}$. Hence $u x \leq v x$ and by Part (1) $x u \leq x v$.

The following definitions are introduced in [3].

Definition 4.4. Let $K$ be a subset of a completely inverse AG-groupoid $S$. Then

(1) $K$ is full, if $E(S) \subseteq K$;

(2) $K$ is self-conjugate, if $u^{-1}(K u) \subseteq K$, for every $u \in K$;

(3) $K$ is inverse closed, if $u \in K \Rightarrow u^{-1} \in K$;

(4) $K$ is normal, if $K$ is full, self-conjugate and inverse closed;

(5) Let $\rho$ be the congruence relation on $S$ as defined in Theorem (2.5). Then restriction $\left.\rho\right|_{E(S)}$ is the trace of $\rho$ to be denoted by tr $\rho$;

(6) The set $k e r \rho=\{u \in S \mid(\exists g \in E(S)) u \rho g\}$.

Example 4.5. Let $S=\{w, x, y, z\}$. Then $(S, *)$ with the Table 5 is an inverse paramedial $A G$-groupoid such that each element is its own inverse. Clearly, $K=\{w, x\}$ is normal in $S$. 


\begin{tabular}{c|cccc}
$*$ & $\mathrm{w}$ & $\mathrm{x}$ & $\mathrm{y}$ & $\mathrm{z}$ \\
\hline $\mathrm{w}$ & $\mathrm{w}$ & $\mathrm{x}$ & $\mathrm{y}$ & $\mathrm{z}$ \\
$\mathrm{x}$ & $\mathrm{x}$ & $\mathrm{w}$ & $\mathrm{z}$ & $\mathrm{y}$ \\
$\mathrm{y}$ & $\mathrm{z}$ & $\mathrm{y}$ & $\mathrm{w}$ & $\mathrm{x}$ \\
$\mathrm{z}$ & $\mathrm{y}$ & $\mathrm{z}$ & $\mathrm{x}$ & $\mathrm{w}$
\end{tabular}

Table 5

Lemma 4.6. Let $\rho$ be a congruence relation on $S$. Then ker $\rho$ is a normal subgroupoid of $S$.

Proof. Since $\rho$ is a congruence relation on $S$, so for any $u, v \in \operatorname{ker} \rho$ there exists $l, m \in$ $E(S)$ such that $u \rho l, v \rho m$. Now uv $l m$, clearly $l m \in E(S)$. So $u v \in k e r \rho$, hence ker $\rho$ is a subgroupoid of $S$. Clearly, $\operatorname{ker} \rho$ is full. Now, let $u \in S$. Then $u^{-1}(k e r \rho \cdot u)=$ $\left\{u^{-1}(v u) \mid v \in k e r \rho\right\}$. Since $v \in \operatorname{ker} \rho$ so there exists $m \in E(S)$ such that $v \rho m$ so, $u^{-1}(v u) \rho u^{-1}(m u)$. Thus

$$
\begin{aligned}
u^{-1}(m u) & =\left(u^{-1} u \cdot u^{-1}\right)(m u) \\
& =\left(u u^{-1}\right)\left(m \cdot u^{-1} u\right) \\
& =(u m)\left(u^{-1} \cdot u u^{-1}\right) \\
& =\left(\left(u^{-1} u\right) \cdot m\right)\left(u^{-1} u\right) \\
& =\left(m \cdot u^{-1} u\right)\left(u^{-1} u\right) \\
& =\left(u^{-1} u \cdot u^{-1} u\right) m \\
& =u^{-1} u \cdot m .
\end{aligned}
$$

Since $u^{-1} u \cdot m \in E(S)$ so $u^{-1}(v u) \in k e r \rho$. Hence $u^{-1}(k e r \rho u) \subseteq k e r \rho$, and thus $k e r \rho$ is self-conjugate subgroupoid of $S$. Also if $u \in k e r \rho$ then $u \rho m$ for some $m \in E(S)$ and $u^{-1} \rho m^{-1}=m$. Hence $u^{-1} \in \operatorname{ker} \rho$, and $\operatorname{ker} \rho$ is inverse closed. Thus $k \operatorname{ker} \rho$ is normal subgroupoid of $S$.

Definition 4.7. [8] Let $K$ be a normal subgroupoid of $S$ and $\tau$ be a congruence on semilattice $E(S)$ such that,

$$
l u \in K, l \tau u^{-1} u \Rightarrow u \in K,
$$

for every $u \in S$ and $l \in E(S)$. Then the pair $(K, \tau)$ is a congruence pair for $S$. In this case, we define a relation $\rho_{(K, \tau)}$ on $S$ by

$$
u \rho(K, \tau) v \Leftrightarrow u^{-1} u \tau v^{-1} v, u v^{-1}, v u^{-1} \in K .
$$

Lemma 4.8. For a congruence pair $(K, \tau)$ for $S$, we have

$$
l(u v) \in K, l \tau u^{-1} u \Rightarrow u v \in K
$$

for any $u, v \in S, l \in E(S)$. 
Proof. Let $u, v \in S, l \in E(S), l(u v) \in K$ and $l \tau u^{-1} u$. Then using the paramedial, medial, left invertive laws and definition of inverse AG-groupoid

$$
\begin{aligned}
l \cdot u v & =l l \cdot u v \\
& =v l \cdot u l \\
& =\left(\left(v v^{-1} \cdot v\right) l\right)(u l) \\
& =\left(l v \cdot v v^{-1}\right)(u l) \\
& =\left(u l \cdot v v^{-1}\right)(l v) \\
& =(u l \cdot l)\left(v v^{-1} \cdot v\right) \\
& =(l l \cdot u)\left(v v^{-1} \cdot v\right) \\
& =(l u)\left(v v^{-1} \cdot v\right) \\
& =\left(l \cdot v v^{-1}\right)(u v)
\end{aligned}
$$

and

$$
\begin{aligned}
(u v)^{-1}(u v) & =\left(u^{-1} v^{-1}\right)(u v) \\
& =\left(u^{-1} u\right)\left(v^{-1} v\right) \tau l\left(v^{-1} v\right) .
\end{aligned}
$$

By above and ( 4. 11 ), we have $u v \in K$.

Definition 4.9. [4] Let $K$ be a full subgroupoid of $S$ and $\tau$ a congruence on $E(S)$ and $\leq$ be the relation as defined in Theorem (3.1) and satisfying the following condition:

(1) For all $u \in S, v \in K, v \leq u$ and $u u^{-1} \tau v v^{-1}$ imply $u \in K$.

We call $(K, \tau)$ a pseudo normal congruence pair for $S$. If, in addition,

(2) For every $u \in K$, there exists $v \in S$ with $v \leq u, u u^{-1} \tau v v^{-1}$ and $v^{-1} \in K$, then $(K, \tau)$ is called normal congruence pair for $S$.

For pseudo normal congruence pair $(K, \tau)$, we define a relation, $\rho_{(K, \tau)}$ as follows:

$$
u \rho_{(K, \tau)} v \Leftrightarrow u v^{-1}, u^{-1} v, v u^{-1}, v^{-1} u \in K, u u^{-1} \cdot v v^{-1} \tau u u^{-1} \tau v v^{-1} .
$$

Lemma 4.10. Let $(K, \tau)$ be a pseudo normal congruence pair of $S, u, v \in S$. If $u \rho_{(K, \tau)} v$ and $v \in K$, then $u \in K$.

Proof. Since $u \rho(K, \tau) v$, so we have $u v^{-1} \in K$ and $u u^{-1} \cdot v v^{-1} \tau v v^{-1}$. Since $v \in K$ and $K$ is full subgroupoid, so $u v^{-1} \cdot v=v v^{-1} \cdot u \in K$. We have to prove that $u v^{-1} \cdot v \leq u$. 
Here

$$
\begin{aligned}
\left(\left(u v^{-1} \cdot v\right)\left(u v^{-1} \cdot v\right)^{-1}\right) u & =\left(\left(u v^{-1} \cdot v\right)\left(u^{-1} v \cdot v^{-1}\right)\right) u \\
& =\left(\left(v v^{-1} \cdot u\right)\left(v^{-1} v \cdot u^{-1}\right)\right) u \\
& =\left(\left(v v^{-1} \cdot v^{-1} v\right)\left(u u^{-1}\right)\right) u \\
& =\left(v v^{-1} \cdot u u^{-1}\right) u \\
& =\left(u u^{-1} \cdot v v^{-1}\right) u \\
& =\left(u u^{-1} \cdot v v^{-1}\right)\left(u u^{-1} \cdot u\right) \\
& =\left(u u^{-1} \cdot u u^{-1}\right)\left(v v^{-1} \cdot u\right) \\
& =u u^{-1}\left(v v^{-1} \cdot u\right) \\
& =u u^{-1}\left(u v^{-1} \cdot v\right) \\
& =v u^{-1}\left(u v^{-1} \cdot u\right) \\
& =\left(v \cdot u v^{-1}\right)\left(u^{-1} u\right) \\
& =\left(u^{-1} u \cdot u v^{-1}\right) v \\
& =\left(v^{-1} u \cdot u u^{-1}\right) v \\
& =\left(\left(u u^{-1} \cdot u\right) v^{-1}\right) v \\
& =u v^{-1} \cdot v .
\end{aligned}
$$

Hence, by ( 3.4 ), it follows that $u v^{-1} \cdot v \leq u$.

Also

$$
\begin{aligned}
\left(u v^{-1} \cdot v\right)\left(u v^{-1} \cdot v\right)^{-1} & =\left(u v^{-1} \cdot v\right)\left(u^{-1} v \cdot v^{-1}\right) \\
& =\left(u v^{-1} \cdot u^{-1} v\right) v v^{-1} \\
& =\left(u u^{-1} \cdot v^{-1} v\right) v v^{-1} \\
& =\left(v v^{-1} \cdot v^{-1} v\right) \cdot u u^{-1} \\
& =v v^{-1} \cdot u u^{-1} \tau u u^{-1} .
\end{aligned}
$$

Hence by Definition (4.9(i)) it follows that $u \in K$.

Theorem 4.11. Let $(K, \tau)$ be a pseudo normal congruence pair for $S$. Then $\rho_{(K, \tau)}$ is a congruence on $S$ with

$$
\operatorname{ker}_{(K, \tau)}=\left\{u \in K \mid(\exists v \in S), v \leq u, u u^{-1} \tau v v^{-1}, v^{-1} \in K\right\}
$$

Proof. Let $\rho_{(K, \tau)}$, be a pseudo normal congruence pair for $S$ as given in ( 4. 12 ) and $\rho=\rho_{(K, \tau)}$. First we show that $\rho$ is compatible, for this assume $u \rho v$ and $w \in S$. Then

$$
u w \cdot(v w)^{-1}=u w \cdot v^{-1} w^{-1}=u v^{-1} \cdot w w^{-1} \subseteq K \cdot E(S) \subseteq K,
$$

By Definition (4.9), for pseudo normal congruence pair and $K$ is full. So, $u w \cdot(v w)^{-1} \in$ $K$. Similarly, $(v w)^{-1} \cdot u w,(u w)^{-1} \cdot v w, v w \cdot(u w)^{-1} \in K$. 
Next,

$$
\begin{aligned}
\left(u w \cdot(u w)^{-1}\right)\left((v w)^{-1} \cdot v w\right) & =\left(u w \cdot(v w)^{-1}\right)\left((u w)^{-1} \cdot v w\right) \\
& =\left(u w \cdot v^{-1} w^{-1}\right)\left(u^{-1} w^{-1} \cdot v w\right) \\
& =\left(u v^{-1} \cdot w w^{-1}\right)\left(u^{-1} v \cdot w^{-1} w\right) \\
& =\left(u v^{-1} \cdot u^{-1} v\right)\left(w w^{-1} \cdot w^{-1} w\right) \\
& =\left(u v^{-1} \cdot u^{-1} v\right)\left(w w^{-1} \cdot w w^{-1}\right) \\
& =\left(u u^{-1} \cdot v^{-1} v\right)\left(w w^{-1} \cdot w w^{-1}\right) \\
& =\left(u u^{-1} \cdot v^{-1} v\right) w w^{-1} \tau u u^{-1} \cdot w w^{-1} \\
\left(u w \cdot(u w)^{-1}\right)\left((v w)^{-1} \cdot v w\right) & \tau\left(u w \cdot(u w)^{-1}\right) .
\end{aligned}
$$

By symmetry, it follows that

$$
\left(u w \cdot(u w)^{-1}\right)\left((v w)^{-1} \cdot v w\right) \quad \tau \quad\left(v w \cdot(v w)^{-1}\right) .
$$

Hence $u w \rho v w$. Thus $\rho$ is right compatible, similarly, $\rho$ is left compatible, thus $\rho$ is compatible.

Now, we have to show that $\rho$ is an equivalence. Since $K$ is full, so $\rho$ is reflexive. Obviously, $\rho$ is symmetric. For transitivity, let $u \rho v, v \rho w$. Then by right compatibility $u w^{-1} \rho v w^{-1}$ and $v w^{-1} \rho w w^{-1}$, since $w w^{-1} \in E(S) \subseteq K$, and $v w^{-1} \rho w w^{-1}$, so $v w^{-1} \in K$ (by Lemma (4.10)). Again $u w^{-1} \rho v w^{-1}$ so again by Lemma (4.10), $u w^{-1} \in K$. Similarly, $u u^{-1} \rho v u^{-1}, v u^{-1} \rho w u^{-1} \Rightarrow w u^{-1} \in K$ (by Lemma (4.10)).

Similarly, by left compatibility $u \rho v, v \rho w$ implies $u^{-1} u \rho u^{-1} v$ and $u^{-1} v \rho u^{-1} w$, and $w^{-1} v \rho w^{-1} w$ so again by Lemma (4.10), we have $u^{-1} w, w^{-1} u \in K$.

Also $u \rho v, v \rho w$ yields

$$
u^{-1} u \cdot v v^{-1} \tau u u^{-1} \tau v v^{-1}, v^{-1} v \cdot w w^{-1} \tau v v^{-1} \tau w w^{-1} .
$$

and by transitivity it follows that $u u^{-1} \tau w w^{-1}$. Moreover,

$$
\begin{aligned}
\left(v v^{-1} \cdot w w^{-1}\right)\left(u u^{-1} \cdot w w^{-1}\right) & =\left(v v^{-1} \cdot u u^{-1}\right)\left(w w^{-1} \cdot w w^{-1}\right) \\
& =\left(v v^{-1} \cdot u u^{-1}\right) w w^{-1} \tau u u^{-1} \cdot w w^{-1},
\end{aligned}
$$

also

$$
\begin{aligned}
\left(v v^{-1} \cdot w w^{-1}\right)\left(u u^{-1} \cdot w w^{-1}\right) & =\left(v v^{-1} \cdot u u^{-1}\right)\left(w w^{-1} \cdot w w^{-1}\right) \\
& =\left(v v^{-1} \cdot u u^{-1}\right) w w^{-1} \tau v v^{-1} \cdot w w^{-1} \tau w w^{-1} .
\end{aligned}
$$

Whence, $u u^{-1} \cdot w w^{-1} \tau w w^{-1}$.

Now, $u w^{-1}, u^{-1} w, w u^{-1}, w^{-1} u \in K, u u^{-1} \cdot w w^{-1} \tau u u^{-1} \tau w w^{-1}$ is equivalent to $u \rho w$. Hence $\rho$ is transitive relation and so is a congruence.

\section{Conclusions}

In this article, the concept of inverse AG-groupoid [4, 7] is extended to paramedial AG-groupoid $S$ that satisfies the paramedial law: $u v \cdot w x=x v \cdot w u$, and various of its properties are investigated. It is proved that inverses in an inverse paramedial AGgroupoid are unique. Congruences, partial order, and compatible partial orders for inverse paramedial AG-groupoid are introduced and investigated. This idea is further proceeded to completely inverse paramedial AG-groupoids. Various notions for completely inverse 
paramedial AG-groupoids are defined and investigated. Furthermore, some congruences on completely inverse paramedial AG-groupoids are introduced and characterized. The concept of separative ordered and completely separative, normal sub-groupoid, pseudo normal congruence pair, and normal congruence pair for the class of completely inverse paramedial AG-groupoids are also introduced and investigated. Various examples are provided for justification of the produced results.

\section{AUthor CONTRIBUtions}

M. Rashad and I. Ahmad developed the theoretical formalism, gave the examples. F. Karaaslan contributed to the final version of the manuscript.

\section{ACKNOWLEDGMENT}

This work is financially supported by the Higher Education Commission, Pakistan (HEC) under the project NRPU-3509.

\section{REFERENCES}

[1] I. Ahmad, A. Ullah , M. Shah, Fuzzy AG-subgroups, Life Science Journal, 9,No. 4 (2012) 3931-3936.

[17] I. Ahmad, Iftikhar Ahmad and M. Rashad, A Study of anti-commutativity in AG-groupoids, Punjab Univ. J. Math. 48, No. 4(2016) 99-109.

[2] M. Bozinovic, P.V. Protic, N. Stevanovic, The natural partial order on the Abel-Grassmann's-groupoids, Filomat 10, (1996) 107-116.

[3] M. Bozinovic, P.V. Protic, N. Stevanovic, Kernel normal system of inverse AG ${ }^{* *}$-groupoid, Quasigroups and related system 17, (2008) 1-8.

[4] W.A. Dudek, R.S. Gigon, CCongruences on completly inverse AG**-groupoid, Quasigroups and related system 20, (2012) 203-209.

[5] F. Hayat, Some basic properties of soft intersection AG-groups, M.Phil thesis, Department of Mathematics, University of Malakand 2016.

[18] M. Khan, I. Ahmad, M. Iqbal, M. Rashad and Amanullah, A Study of Anti-rectangular AG-groupoids, Punjab Univ. j. math. 51),No. 12 (2019) 71-92.

[6] M. Kazim, M. Naseeruddin, On almost semigroups, Alig. Bull. Math 2, (1972) 1-7.

[7] Q. Mushtaq, Iqbal. Partial ordering and congruences on LA-semigroup, Indian J. Pure App. Math. 22, No. 4(1991) 331-336.

[8] P.V. Protic, M. Bozinovic, Some congruences on an $A G^{* *}$-groupoid, Filomat 9, No. 4 (1995) 879-886.

[9] Protic P.V. Congruences on an inverse $A G^{* *}$-groupoid via the natural partial order, Quasigroups and related system 17, (2009) 283-290.

[10] M. Rashad, Investigation and classification of some new classes of AG-groupoids, Ph.D. thesis, Department of Mathematics, University of Malakand Chakdara Dir (L), 2015.

[11] M. Rashad, A. Ullah, I. Ahmad, Modulo matrix AG-groupoids and modulo AG-groups, Mehran University Research Journal of Engineering Technology 35,No. 1 (2016) 63-70.

[19] M. Rashad and I. Ahmad, A note on unar LA-semigroup, Punjab Univ. j. math. 50, No.3 (2018) 113-121.

[20] M. Rashad, I. Ahmad, Amanullah and M. Shah, A Study on Cheban Abel-Grassmann's Groupoids, Punjab Univ. j. math. 51, No. 2(2019) 79-90

[12] M. Shah,A theoretical and computational investigation of AG-groups, $\mathrm{PhD}$ thesis, Quaid-i-Azam University Islamabad, Pakistan (2012).

[13] A. Ullah, M. Rashad, I. Ahmad, Shah M. On modulo AG-groupoids, Journal of Advances in Mathematics (JAM) 8,No. 3 (2014) 1606-1613.

[14] A. Ullah, I. Ahmad, M. Shah, On the equal-height elements of fuzzy AG-subgroups, Life Science Journal, 10, No. 4(2013) 3143-3146.

[15] A. Ullah, Fuzzy AG-subgroups, PhD thesis, Department of Mathematics, University of Malakand 2016. 
[16] A. Ullah, M. Rashad, I. Ahmad, Abel-Grassmann groupoids of modulo matrices, Mehran University Research Journal of Engineering and Technology, 35, No. 1 (2016) 63-70.

[21] F. Yousafzai, M. Khan, K. P. Shum and K. Hila, Congruences Induced by Certain Relations on AG**groupoids, U.P.B. Sci. Bull., Series A, 82, No. 2 (2020) 83-100. 